\title{
RESPIRACÃO MICROBIANA DO SOLO SOB DOSES DE GLYPHOSATE E DE IMAZAPYR ${ }^{1}$
}

\author{
ADAILSON P. DE SOUZA ${ }^{2}$, FRANCISCO A. FERREIRA ${ }^{3}$, ANTÔNIO A. DA SILVA ${ }^{3}$, \\ ANTÔNIO A. CARDOSO 3 e HUGO A. RUIZ
}

\section{RESUMO}

A degradação microbiana dos herbicidas no solo é influenciada por diversos fatores, dentre esses as características físicas e químicas do solo e da própria molécula, que agem continuamente determinando a sua magnitude. Em razão da grande variabilidade de respostas apresentadas por essas moléculas no solo e das poucas informações sobre o seu comportamento em ambientes tropicais, no que diz respeito a sua degradação, é que foi realizada uma série de experimentos, em laboratório, objetivando verificar a resposta da atividade microbiana do solo, pelo método da evolução do $\mathrm{CO}_{2}$ do solo, sob doses crescentes do glyphosate e do imazapyr (0; 4; 8 e $12 \mathrm{~L} \mathrm{ha}^{-1}$ do produto comercial Roundup S.Aq.C. e Arsenal 250 S.Aq.C., respectivamente), em solos de diferentes texturas e composição química, sob três conteúdos de umidade (40, 70 e $100 \%$ do equivalente de umidade). Os resultados obtidos permitiram concluir que: a) a microbiota do solo é capaz de utilizar o glyphosate e o imazapyr como fontes de carbono; b) a umidade do solo influencia a capacidade de os microrganismos degradarem o glyphosate e o imazapyr; c) a umidade de $40 \%$, nos solos avaliados, proporcionou as menores respostas da atividade dos microrganismos, independentemente das doses dos herbicidas; e d) o efeito das doses do imazapyr sobre a atividade microbiana do solo é influenciado pelas características físicas e químicas dos solos.

Palavras chave: Atividade microbiana, biodegradação, herbicida.

\section{ABSTRACT \\ Microbial respiration under doses of glyphosate and imazapyr}

The microbial degradation of herbicides in soil is affected by several factors such as the chemical and physical characteristics of the soil and the proper molecule which act continuously determining the degradation magnitude. Considering the large variability of the responses presented by these molecules in the soil as well as the scarce information about their behavior in tropical environments relatively to their degradation, a series of experiments was carried out under laboratory conditions aiming to verify the response of soil microbial activity by the soil$\mathrm{CO}_{2}$ evolution method under increasing doses of glyphosate and imazapyr $\left(0 ; 4 ; 8\right.$ and $12 \mathrm{~L} \mathrm{ha}^{-1}$ of the commercial product Roundup S.Aq.C. and Arsenal 250 S.Aq.C., respectively) in soils with different textures and chemical composition under three moisture contents $(40 ; 70$ and $100 \%$ of the moisture equivalent). The obtained results allowed to conclude that: a) the soil macrobiota is able to use the glyphosate and imazapyr as carbon sources; b) the soil moisture affects the microorganisms capacity to degrade the glyphosate and imazapyr; c) the $40 \%$ moisture in

\footnotetext{
${ }^{1}$ Recebido para publicação em 06/06/98 e na forma revisada em 06/08/99.

${ }^{2}$ Prof $^{\circ}$ Adjunto do Dept ${ }^{\circ}$ de Solos e Eng. Rural da UFPB. CEP: 58397-000, Areia/PB.

${ }^{3}$ Prof $^{\circ}$ Adjunto do Dept ${ }^{\circ}$ de Fitotecnia da UFV. CEP: 36571-000, Viçosa/MG.

${ }^{4}$ Prof $^{\circ}$ Adjunto do Dept ${ }^{\circ}$ de Solos da UFV. CEP: 36571-000, Viçosa/MG.
} 
the studied soils caused the lowest responses of microorganism activities independently of the herbicide doses; d) the effect of imazapyr doses on the soil microbial activity is

\section{INTRODUÇÃO}

A atividade microbiana do solo é um importante fator que influencia o comportamento de herbicidas no ambiente. A taxa de degradação dessas moléculas no solo é função de múltiplos fatores, em que a densidade da população microbiana, as propriedades físico-químicas, e a biodisponibilidade das moléculas e fatores como $\mathrm{pH}$, temperatura, umidade, nutrientes etc., agem continuamente de forma isolada e, ou, interagindo entre si (Rao et al., 1993; Aislabie \& Lloyd-Jones, 1995).

A complexa interação entre microrganismos, substratos e os constituintes do solo pode alterar a capacidade de degradação dessas moléculas. A biodegradação de um herbicida no solo é função da capacidade adsortiva do solo. Quando o herbicida atinge o solo, a adsorção na superfície dos colóides pode reduzir a sua concentração no meio aquoso em níveis que não induziriam os microrganismos a produzir as enzimas necessárias à sua degradação (Madsen, 1991). Também a adsorção poderia reduzir a concentração a níveis que não seriam tóxicos aos microrganismos, o que resultaria no aumento da degradação dessas moléculas (Van Loosdrecht et al., 1990). Em ambos os casos, o conteúdo de água influencia diretamente esse fenômeno (Flint \& Witt, 1997). Em geral, o aumento do conteúdo de água no solo resulta no aumento das taxas de biodegradação (Walker, 1987).

A degradação do glyphosate no solo ocorre predominantemente pela atividade microbiana (Rueppel et al., 1977). Normalmente, é observada uma taxa de decomposição inicialmente rápida, seguida por prolongada e lenta decomposição (Moshier \& Penner, 1978). Esse comportamento reflete a facilidade com que a molécula é metabolizada pela microbiota do solo influenced by the soil chemical and physical characteristics.

Key words: Microbial activity, biodegradation, herbicide.

(Torstensson \& Aamisepp, 1977; Dick \& Quinn, 1995). Embora o glyphosate seja facilmente degradado pelos microrganismos do solo, tem-se observado uma faixa de variação na meia-vida da molécula de poucos dias a anos (Heinonen-Tanski, 1989; Roy et al., 1989; Newton et al., 1994), o que demonstra um estreito relacionamento com os fatores edáficos e climáticos (Torstensson et al., 1989; Souza et al., 1996).

O metabolismo das imidazolinonas, grupo a qual pertence o imazapyr, é caracterizado por ser lento, porém, de degradação contínua (Mangels, 1991). Segundo o autor, estudos têm demonstrado que o imazapyr apresenta, quando incubado a 22 $24^{\circ} \mathrm{C}$, uma meia-vida de 17 e 37 meses, em solos de textura arenosa e argilosa, respectivamente, e que sob temperaturas de 25 e $35^{\circ} \mathrm{C}$, a meia vida é de 5,9 e 7,5 anos, respectivamente.

A taxa de evolução de $\mathrm{CO}_{2}$ do solo tem sido utilizada como índice da atividade microbiana, do tamanho da biomassa do solo e, ainda, do metabolismo do carbono lábil do solo. Os métodos respirométricos estático e dinâmico são os dois mais utilizados nas pesquisas em campo e laboratório (Parkinson et al., 1971; Sakamoto \& Yoshida, 1988). Uma ampla discussão sobre o uso de dados respirométricos, para avaliar a cinética de biodegradação de compostos orgânicos no solo, é apresentada por Smets et al. (1996). No método dinâmico, um fluxo contínuo de ar passa sobre o solo e a quantidade de $\mathrm{CO}_{2}$ liberada pela amostra é quantificada. Esse método tem-se mostrado conveniente, rápido e de boa precisão nas pesquisas com biodegradação de herbicidas (Skipper et al., 1996).

Em razão da grande variabilidade de respostas apresentadas por essas moléculas no solo e das poucas informações sobre o seu comportamento em ambientes tropicais, o 
presente trabalho foi realizado com o objetivo de verificar a respiração da microbiota de dois solos, sob três conteúdos de umidade, submetidos a doses dos herbicidas glyphosate e imazapyr.

\section{MATERIAL E MÉTODOS}

Os experimentos foram conduzidos no Laboratório de Herbicida no Solo do
Departamento de Fitotecnia da Universidade Federal de Viçosa, MG, com solos latossólicos provenientes das subestações da CEMIG, dos municípios de Viçosa e Sabará, MG. As amostras foram coletadas na camada de 0 a $20 \mathrm{~cm}$ de profundidade, secas ao ar e peneiradas em peneira de 4,0 e $2,0 \mathrm{~mm}$ de abertura de malha, para os ensaios de respiração microbiana e caracterização química, física e mineralógica (Tabela 1), respectivamente.

TABELA 1. Composição química, física e mineralógica das amostras dos solos.

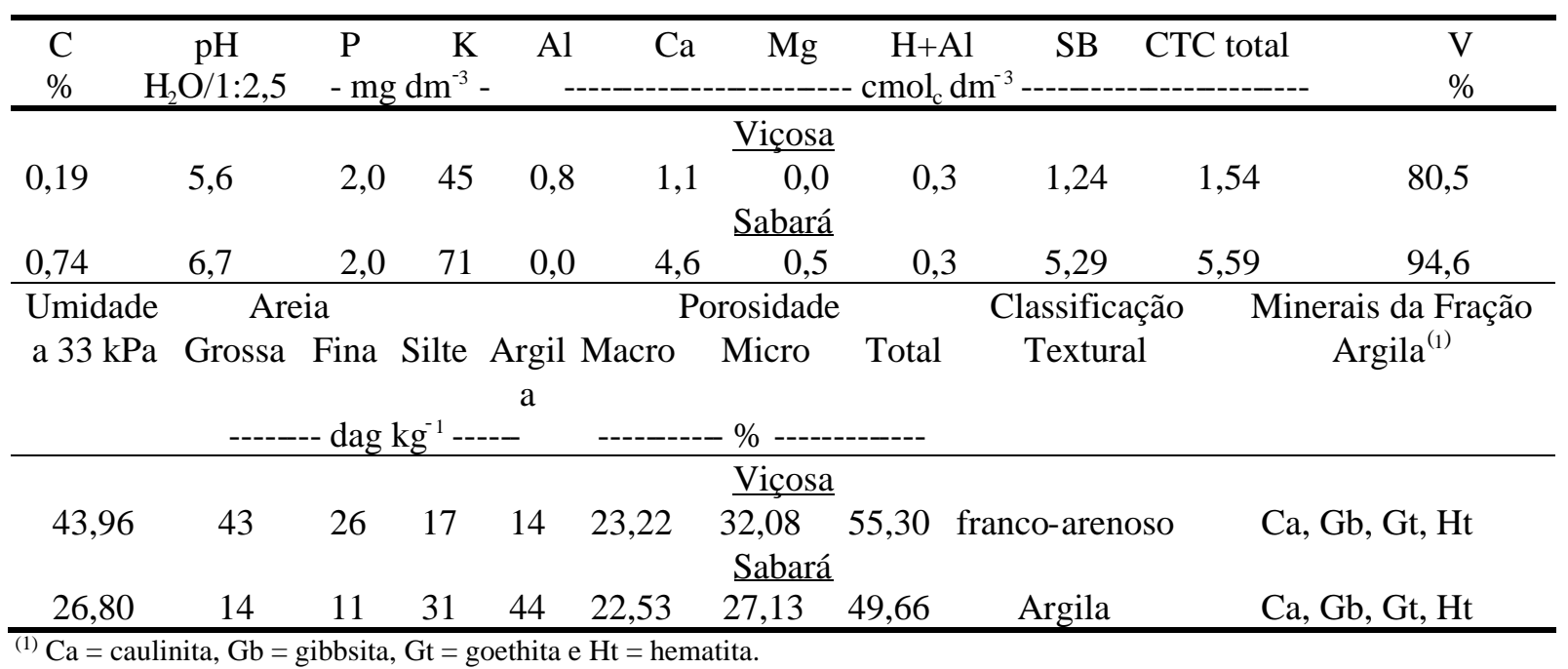

A respiração da microbiota do solo, à temperatura ambiente $\left(23^{\circ} \mathrm{C} \pm 5\right)$, foi determinada pelo método da captura, em solução de $\mathrm{NaOH}$, do $\mathrm{CO}_{2}$ evoluído do solo, sob um sistema de fluxo contínuo (Curl \& Rodriguez-Kabana, 1972). Cada herbicida, e material de solo, foi estudado, separadamente, em delineamento inteiramente casualizado com as parcelas subdivididas quanto a época de avaliação, com três repetições. Os tratamentos nas parcelas foram dispostos em um esquema fatorial $4 \times 3$, correspondendo o primeiro fator às doses comerciais do herbicida $(0 ; 4 ; 8 \mathrm{e}$ $\left.12 \mathrm{~L} \mathrm{ha}^{-1}\right) \mathrm{e}$, o segundo, à umidade do solo (40; 70 e $100 \%$ do equivalente de umidade). Os tratamentos das subparcelas corresponderam às épocas de avaliação (10; 20; 30 e 40 dias).

De cada material de solo, retirou-se uma porção de $100 \mathrm{~g}$, que foi colocada em frascos erlenmeyer de $250 \mathrm{ml}$. Em seguida, nos tratamentos correspondentes, foi feita a aplicação de água necessária para alcançar a umidade desejada. Após uma hora, foi feita a aplicação do herbicida, utilizando-se uma seringa de $5 \mathrm{ml}$, com a extremidade da agulha em forma de leque, o que permitiu a pulverização do herbicida, superficialmente, sobre a amostra de solo nas dosagens correspondentes. Em seguida, os frascos foram conectados a um sistema de fluxo contínuo de ar livre de $\mathrm{CO}_{2}$ (Figura 1), em que o ar que passava pela amostra de solo (Figura $1 \mathrm{H}$ ) era canalizado para um frasco erlenmeyer de $250 \mathrm{ml}$, contendo $100 \mathrm{ml}$ de uma solução de $\mathrm{NaOH}$ a 0,25 M (Figura 1). Nesse frasco, contendo o $\mathrm{NaOH}$, foi ajustada a vazão de fluxo de ar a $2 \mathrm{ml} \mathrm{min}{ }^{-1}( \pm 0,2)$. As titulações foram realizadas aos 10, 20, 30, e 40 dias após iniciado o fluxo de 
ar. A cada titulação, os frascos erlenmeyers contendo o $\mathrm{NaOH}$ foram vedados e substituídos por novos, contendo a mesma solução. $\mathrm{O} \mathrm{NaOH}$ que não reagiu foi titulado com solução de $\mathrm{HCl}$ 0,1 M, previamente padronizado com solução de carbonato de sódio $\left(\mathrm{Na}_{2} \mathrm{CO}_{3}\right) 0,05 \mathrm{M}$.

A quantidade de $\mathrm{CO}_{2}$ evoluído de cada amostra foi calculada em $\mathrm{Cmol}_{c}$ de $\mathrm{CO}_{2}$ por $\mathrm{dm}^{3}$ de solo, segundo a fórmula:

$$
\mathrm{Cmol}_{\mathrm{c}} \mathrm{CO}_{2} \mathrm{dm}^{-3} \text { de solo }=\frac{(\mathrm{B}-\mathrm{T}) \times \mathrm{N} \times \mathrm{f} \times \mathrm{V}}{\mathrm{AT}}
$$

em que:

$\mathrm{B}=$ titulação do branco, $\mathrm{ml}$ do $\mathrm{HCl}$;

$\mathrm{T}=$ titulação do tratamento, $\mathrm{ml}$ do $\mathrm{HCl}$ no solo;
$\mathrm{N}=$ normalidade de $\mathrm{HCl}\left(\mathrm{eq}_{-} \mathrm{g} \mathrm{L}^{-1}\right)$;

$\mathrm{f} \quad=0,9998$ (fator de correção do ácido);

$\mathrm{V}=$ volume de $\mathrm{NaOH}$ usado na captura do $\mathrm{CO}_{2}$ $(\mathrm{ml}) ; \mathrm{e}$

$\mathrm{AT}=$ alíquota a ser titulada $(\mathrm{ml})$.

A análise de variância dos dados foi realizada de acordo com o modelo para experimentos em parcelas subdivididas, em que as somas dos quadrados dos efeitos principais (dose, umidade e suas interações) e secundários (época e interações com dose e umidade) foram desdobrados em regressão, segundo sua significância pelo teste $\mathrm{F}$.

\section{$\mathrm{B}$}

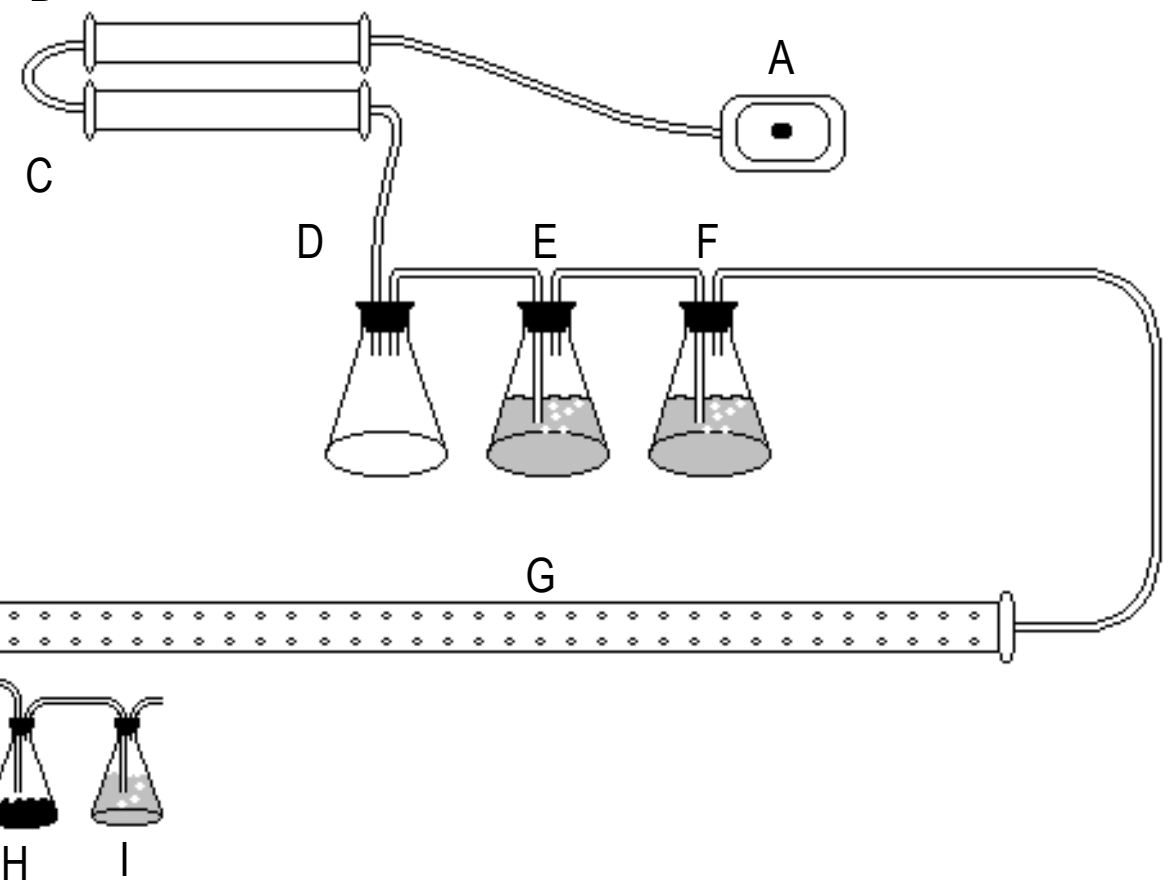

FIGURA 1. Representação esquemática do sistema utilizado na determinação da evolução do $\mathrm{CO}_{2}$ do solo. A, bomba; B, $\mathrm{CaSO}_{4}$; C, NaOH; D, refluxo; E, solução de $\mathrm{NaOH} 1 \mathrm{M}(2 / 3 \mathrm{~L}) ; \mathrm{F}$, solução de $\mathrm{Ba}(\mathrm{OH})_{2} 1 \%$ (2/3 L); G, linha de distribuição; H, amostra de solo (100 g); e I, solução de $\mathrm{NaOH} 0,25 \mathrm{M}(100 \mathrm{ml})$.

\section{RESULTADOS E DISCUSSÃO}

Os resultados da respiração da biota, medidos pela quantidade de $\mathrm{CO}_{2}$ liberada, em função das doses e umidades dos solos, estão apresentados na Figura 2.

As doses dos herbicidas e o conteúdo de umidade apresentaram efeitos bastante variados sobre a respiração microbiana dos solos estudados 
(Figura 2). Nos experimentos com o glyphosate, verificou-se que as menores taxas de respiração foram observadas na dose zero e com o menor conteúdo de água no solo (40\% do equivalente de umidade). Esse resultado demonstrou que os microrganismos são capazes de utilizar o glyphosate como fonte de carbono e que a umidade do solo determina a magnitude desta resposta. Esse comportamento é explicado pelo efeito significativo da interação dose (D) e umidade (U). A significância dessa interação indicou a dependência da respiração microbiana quando tratado com glyphosate e do conteúdo de água do solo, o que também tem sido constatado em outros trabalhos com herbicidas (Choi et al., 1988; Flint \& Witt, 1997).

O metabolismo do glyphosate no solo tem sido amplamente estudado. Segundo Sprankle et al. (1975), a ação dos microrganismos é o principal fator responsável por sua degradação no solo. Heinonen-Tanski (1989), Wardle \& Parkinson (1990) e Wardle et al. (1994) observaram que essa degradação está estreitamente relacionada à taxa de $\mathrm{CO}_{2}$ liberada do solo. A quebra da ligação $\mathrm{C}-\mathrm{P}$ da molécula é apresentada como a primeira etapa desse processo (Kishore \& Jacob, 1987; Pipke et al., 1987; Liu et al., 1991; Dick \& Quinn, 1995), não havendo, entretanto, evidências de sua completa mineralização. Torstensson \& Aamisepp (1977) constataram que o glyphosate não era diretamente utilizado como fonte de energia para a população microbiana, sendo apenas co-metabilizado. Maiores evidências sobre o processo de cometabolismo foram obtidos por Torstensson \& Stenström (1986), ao observarem que a produção de enzimas microbianas necessárias para a quebra do glyphosate não era induzida pelo próprio herbicida, mas por outros substratos orgânicos. Assim, segundo Torstensson et al. (1989), o processo co-metabólico do glyphosate depende da própria atividade global da microbiota do solo, embora essa venha a ser induzida pelo próprio herbicida.

Ainda na Figura 2, verificou-se que o aumento das doses do imazapyr apresentou respostas muito distintas entre os solos estudados. No solo de Viçosa, embora o aumento das doses tenha promovido um comportamento cúbico no ajuste da superfície de resposta, a dose de $12 \mathrm{~L} \mathrm{ha}^{-1}$ resultou num aumento crescente na respiração da biota, com o aumento da umidade do solo. No solo de Sabará, há um comportamento quadrático com o aumento das doses, refletindo nas maiores doses um efeito inibidor do imazapyr sobre a respiração microbiana, nas umidades de 40 e $100 \%$. Pelas diferenças de respostas apresentadas entre os solos, verificou-se que as características químicas e físicas influenciaram significativamente na respiração da biota. O resultado justifica, em parte, as diferenças de persistência dessa molécula em diferentes classes de solo (Nicholls, 1988; Loux \& Reese, 1993; Michael \& Neary, 1993). Segundo Skipper et al. (1996), o potencial biodegradativo do solo é influenciado por numerosos fatores, dentre eles as próprias condições de laboratório.

Nas Figuras 3 e 4, são apresentadas as taxas de $\mathrm{CO}_{2}$ liberadas dos solos de Viçosa e Sabará, nas diferentes épocas de avaliação, para cada dose dos herbicidas glyphosate (Figura 3) e imazapyr (Figura 4) e nas diferentes umidades do solo. Observou-se, por estes resultados, que, independente da dose aplicada dos herbicidas e dos solos estudados, a umidade de $40 \%$ resultou nas menores taxas de evolução de $\mathrm{CO}_{2}$, exceção observada apenas no tratamento $12 \mathrm{~L} \mathrm{ha}^{-1}$ aos 40 dias (Figura 3 h). Segundo Paul \& Clark (1989), a atividade microbiana do solo é fortemente regulada pelo conteúdo de água do solo, seja por ser um componente indispensável ao protoplasma celular, seja por modificar as trocas gasosas do solo, seja por determinar a solubilidade e a disponibilidade dos nutrientes. No experimento com o glyphosate, a umidade de $70 \%$ apresentou as melhores respostas dos microrganismos nas doses de 4, 8 e $12 \mathrm{~L} \mathrm{ha}^{-1}$, no solo de Sabará (Figura $3 \mathrm{f}, \mathrm{g}, \mathrm{h}$ ). Verificou-se, na Figura 4, que com o imazapyr as maiores respostas foram obtidas na umidade de $100 \%$ no solo de Viçosa e de $70 \%$ no solo de Sabará. 


\section{Glyphosate}

a

$P=-0,332-0,152 D+0,0295^{*} U-0,000114^{* *} U^{2}+0,00543 D U-$ $-0,0000409 \mathrm{DU}^{2}$

$R^{2}=0,9469$

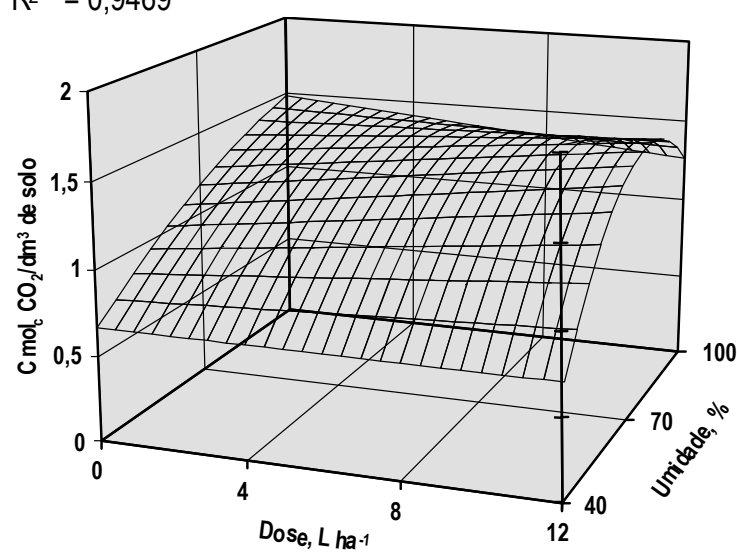

$P=-0,288+0,0845^{\circ} \mathrm{D}-0,0033^{\circ} \mathrm{D}^{2}+0,0449^{* *} \mathrm{U}-0,000269^{* *} U^{2}-$ $-0,0004810 \mathrm{DU}$

$R^{2}=0,9489$

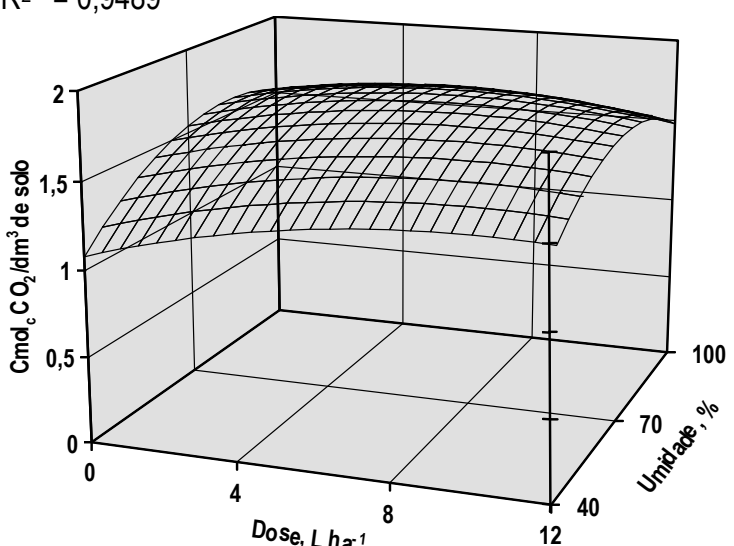

\section{Imazapyr}

b

$\nexists=-1,678+0,133^{\circ} D-0,0406 D^{2}+0,00314^{* *} D^{3}+0,0859^{* *} U-$ $-0,000471^{* *} U^{2}+0,000364 \mathrm{DU}+0,0000179 \mathrm{DU} 2-$ $-0,00023^{*} D^{2} U$

$R^{2}=0,9964$

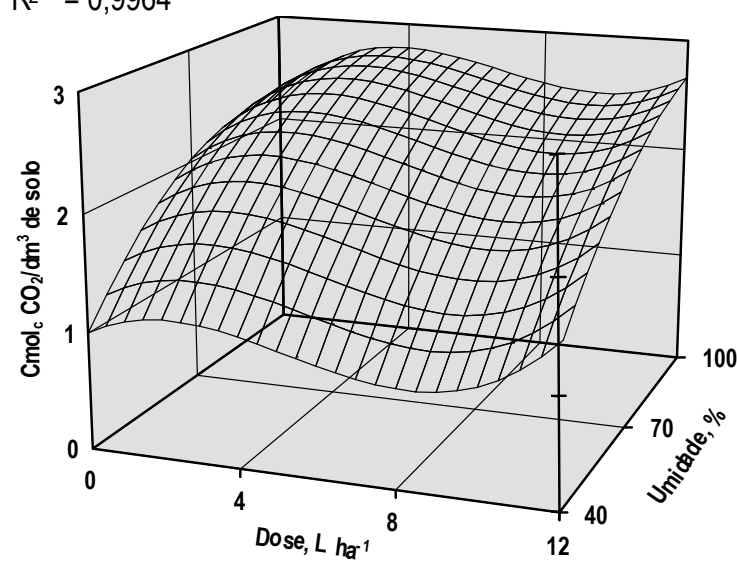

d

$$
\begin{aligned}
P= & -2,921-0,667 D-0,0187^{*} D^{2}+0,175^{*} U-0,00119^{* *} U^{2}+ \\
& +0,0295 D U-0,000207^{* *} D^{2} \\
R^{2} & =0,9355
\end{aligned}
$$

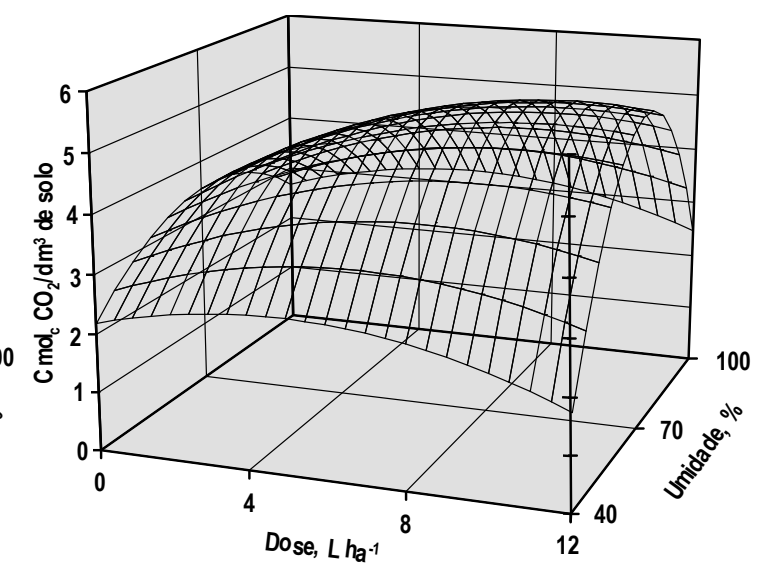

FIGURA 2. $\mathrm{CO}_{2}$ liberado pela respiração biótica dos solos de Viçosa (a e b) e Sabará (c e d), em função das diferentes doses do glyphosate e do imazapyr e das umidades do solo. 
a

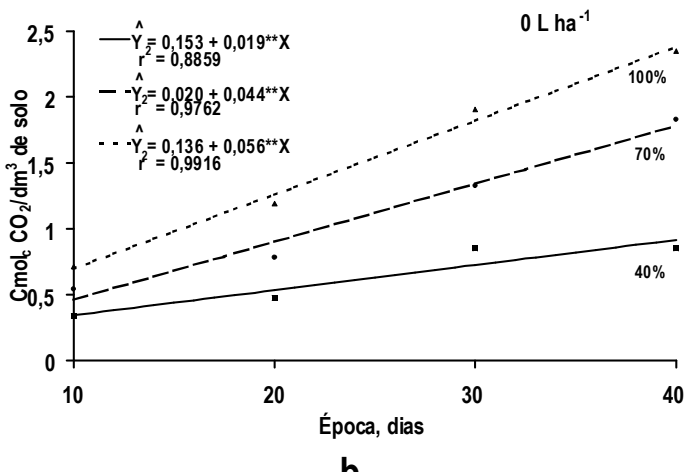

b
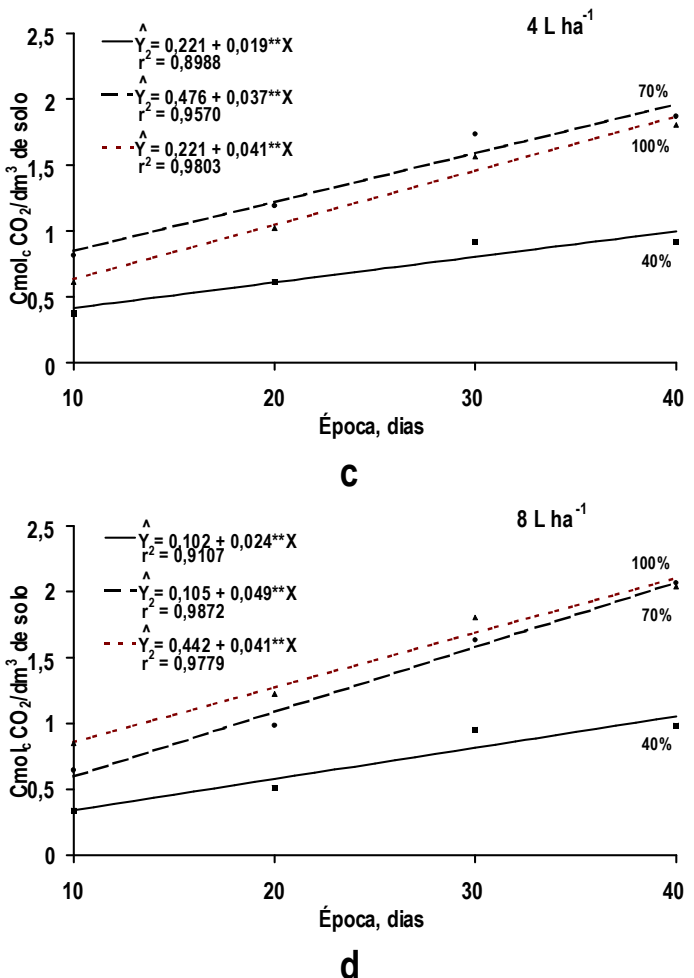

d

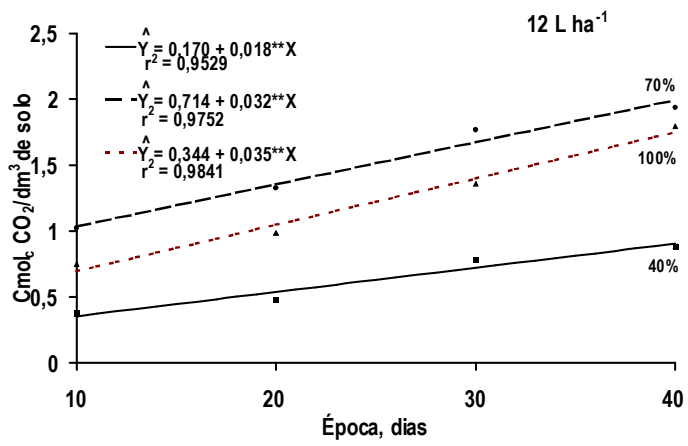

e
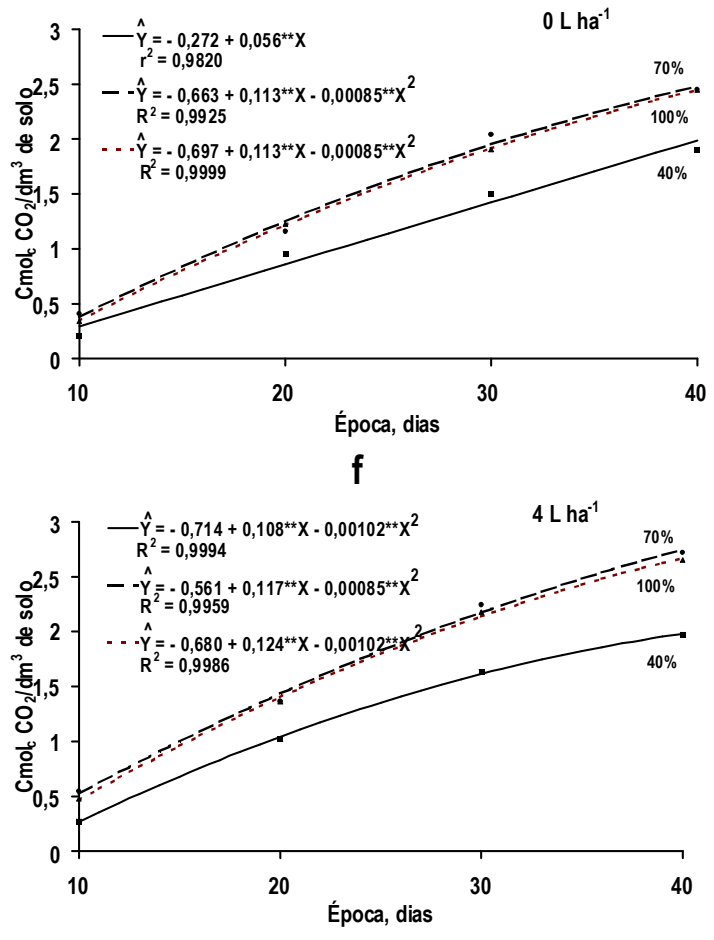

$\mathrm{g}$

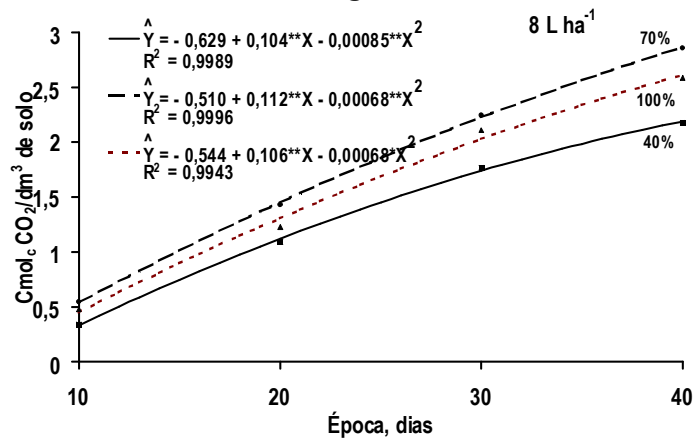

h

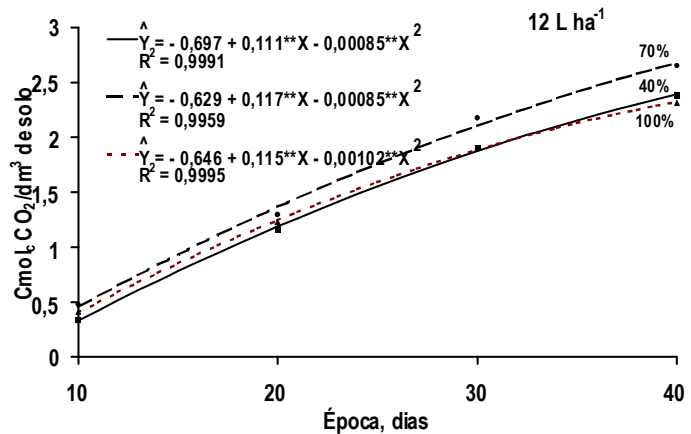

FIGURA 3. $\mathrm{CO}_{2}$ liberado pela atividade microbiana dos solos de Viçosa (a, b, c e d) e Sabará (e, f, g e h), nas diferentes umidades do solo (40; 70 e 100\% do equivalente de umidade), e nas doses 0 ; $4 ; 8$ e $12 \mathrm{~L} \mathrm{ha}^{-1}$ de glyphosate. 
a

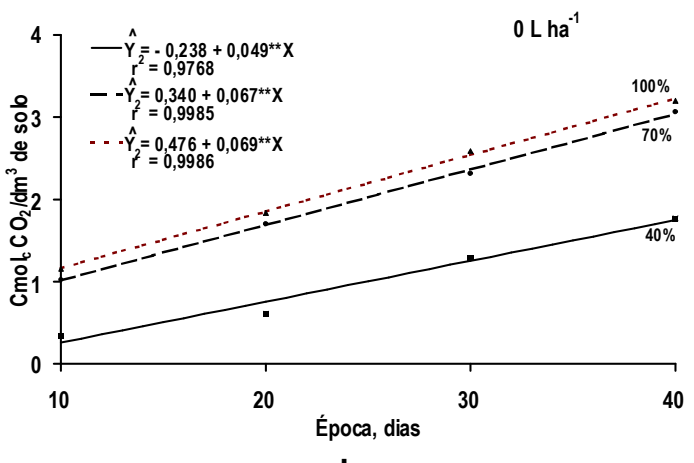

b

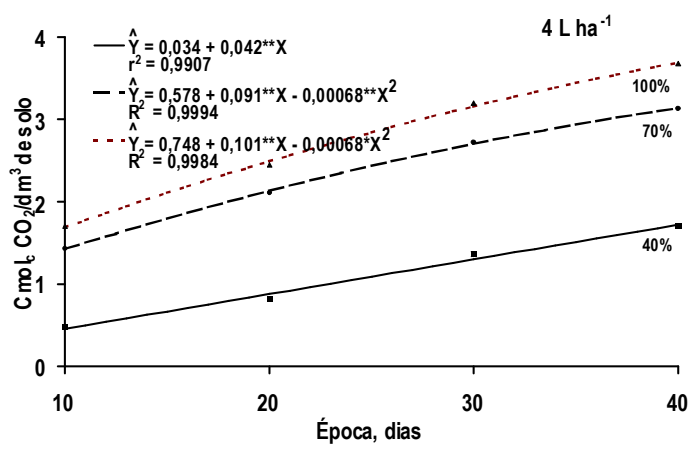

C

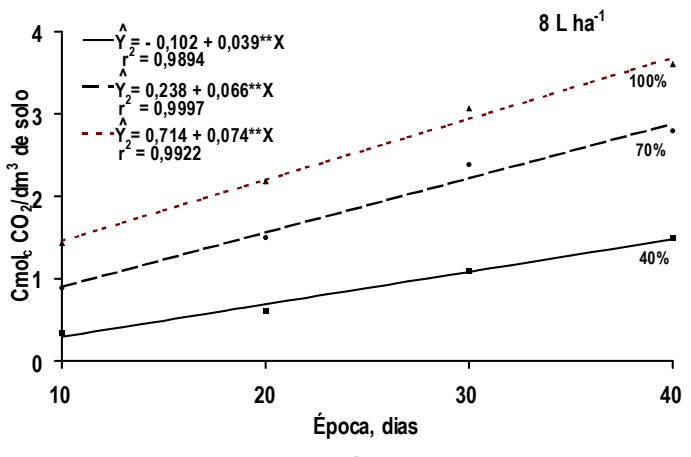

d

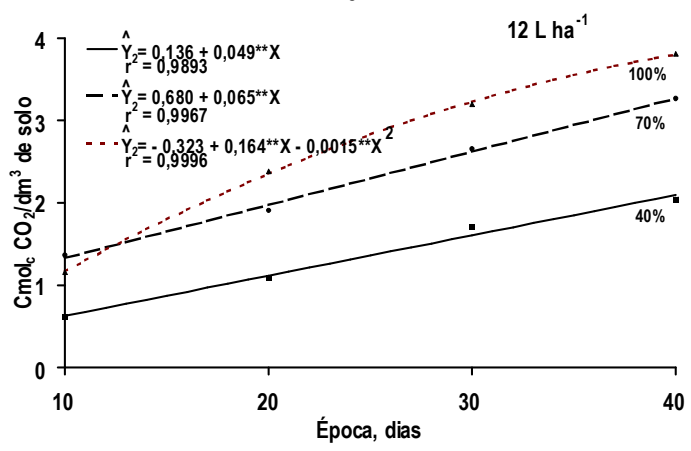

e

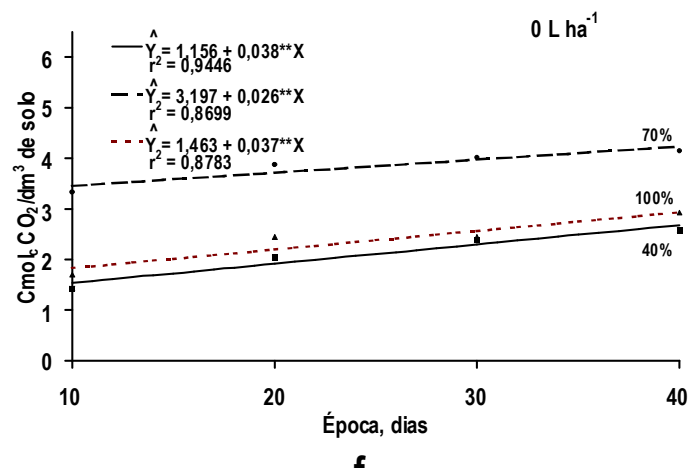

f

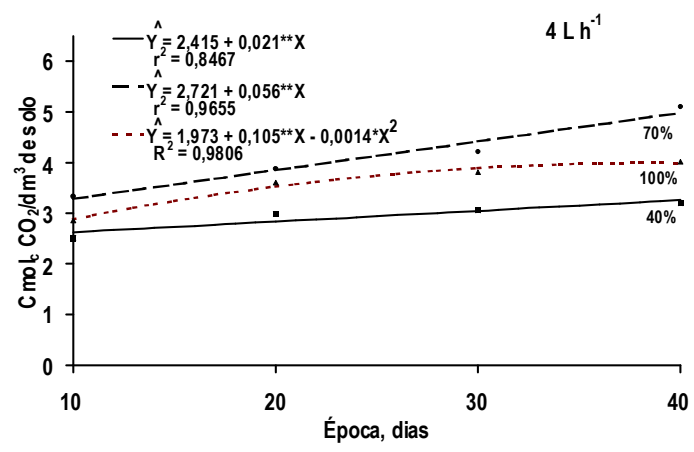

g

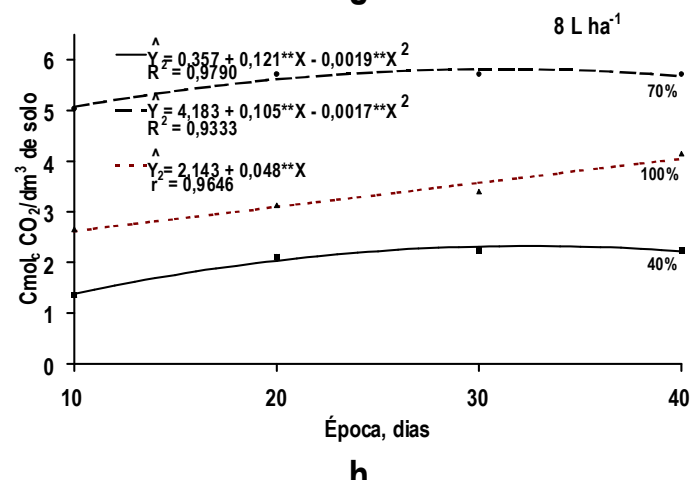

$h$

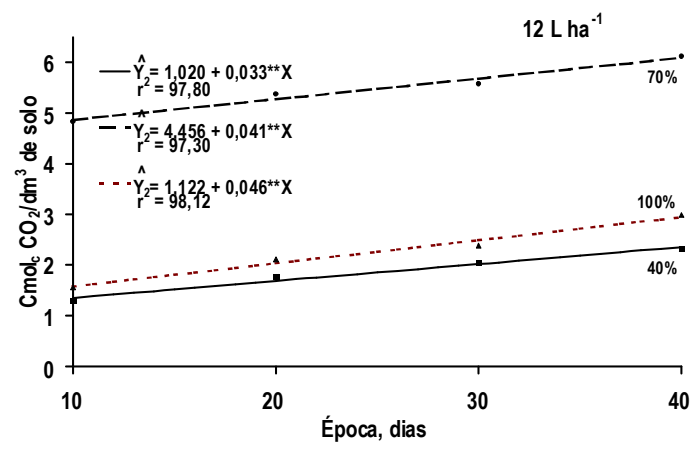

FIGURA 4. $\mathrm{CO}_{2}$ liberado pela atividade microbiana dos solos de Viçosa (a, b, c e d) e Sabará (e, f, g e h), nas diferentes umidades do solo (40, 70 e 100\% do equivalente de umidade), e nas doses $0 ; 4 ; 8$ e $12 \mathrm{~L} \mathrm{ha}^{-1}$ de imazapyr. 
Essa diferença nas taxas de $\mathrm{CO}_{2}$ reflete um estreito relacionamento das características físicas e químicas desses solos com a umidade e com a capacidade dos microrganismos em degradar esta molécula. No solo de Viçosa, de textura mais arenosa, é observado um maior percentual de macro e microporos $(23,22$ e $32,08 \%$, respectivamente), o que facilitaria uma maior aeração, mesmo com a umidade a $100 \%$ do equivalente de umidade. A atividade global da microbiota, predominantemente aeróbica nessas condições, seria favorecida pela maior disponibilidade de nutrientes, inclusive do próprio imazapyr, o que justifica as maiores respostas nas dosagens de 4,8 e $12 \mathrm{~L} \mathrm{ha}^{-1}$. Segundo Pivetz \& Steenhuis (1995), os macroporos podem apresentar um ambiente favorável à biodegradação, por causa do grande fornecimento de oxigênio, nutrientes e substratos orgânicos, e altas populações de microrganismos. No solo de Sabará, de textura mais argilosa e de menor porosidade total, com a umidade do solo a $100 \%$, ocorreu saturação da microporosidade e parte da macroporosidade, o que reduziu as trocas gasosas nesse solo, comprometendo, assim, a atividade dos microrganismos aeróbicos e a degradação do imazapyr (Figura 2 d, Figura 4 e, f, g, h).

Segundo Ismail \& Ahmad (1994), o estreito relacionamento entre a degradação do imazapyr e a variação da umidade do solo decorre da fraca adsorção dessa molécula às partículas do solo. Sob condições de maior umidade, a molécula estaria mais facilmente disponível aos microrganismos do solo. Felsot \& Shelton (1993) afirmam que a umidade do solo é fator-chave na expressão do aumento da biodegradação, não somente pela a influência da umidade na fisiologia da degradação, mas também pelas taxas de dessorção dessas moléculas da superfície dos constituintes do solo.

\section{LITERATURA CITADA}

AISLABIE, J., LLOYD-JONES, G. A review of bacterial degradation of pesticides. Aust. j. soil res., v.33, n.6, p.925-942, 1995.
CHOI, J.S.T., FERMANIAN, T.W., WEHNER, D.J. et al. Effect of temperature, moisture, and soil texture on DCPA degradation. Agron. j., v.80, n.1, p.108-113, 1988.

CURL, E.A., RODRIGUEZ-KABANA, R. Microbial interactions. In: WILKINSON, R.E. (Ed.). Research methods in weed science., Atlanta: Southern Weed Science Society, 1972. p.162-194

DICK, R.E., QUINN, J.P. Glyphosate-degrading isolates from environmentall samples: occurrence and pathways of degradation. Appl. microbiol. biotechnol., v.43, n.3, p.545-550, 1995.

FELSOT, A.S., SHELTON, D.R. Enhanced biodegradation of soil pesticides: interactions between physicochemical processes and microbial ecology. In: LINN, D.M., CARSKI, T.H., BRUSSEAU, M.L. et al. (Eds.). Sorption and degradation of pesticides and organic chemicals in soil. Madison, Wisconsin, USA: SSSA Inc., 1993. p.227-251. (SSSA Special publication, 32)

FLINT, J.L., WITT, W.W. Microbial degradation of imazaquin and imazethapyr. Weed sci., v.45, n.4, p.586-591, 1997.

HEINONEN-TANSKI, $\mathrm{H}$. The effect of temperature and liming on the degradation of glyphosate in two arctic forest soils. Soil biol. biochem., v.21, n.2, p.313-317, 1989.

ISMAIL, B.S., AHMAD, A.R. Attenuation of the herbicidal activities of glufosinateammonium and imazapyr in two soils. Agric. ecosyst. environ., v.47, n.2, p.279285, 1994.

KISHORE, G.H., JACOB, G.S. Degradation of glyphosate by Pseudomonas sp. pG2982 via sarcosine intermediate. J. biol. chem., v.262, n.25, p.12164-12168, 1987. 
LIU, C.-M., McLEAN, P.A., SOOKDEO, C.C. et al. Degradation of the herbicide glyphosate by members of the family Rhizobiacae. Appl. environ. microbiol., v.57, n.7, p.1799-1804, 1991.

LOUX, M.M., REESE, K.D. Effect of soil type and $\mathrm{pH}$ on persistence and carryover of imidazolinone herbicides. Weed Technol., v.7, n.2, p.452-458, 1993.

MADSEN, E.L. Determining in situ biodegradation: facts and challenges. Environ. sci. technol., v.25, n.1, p.16631673, 1991.

MANGELS, G. Behavior of the imidazolinone herbicides in soil - A review of the literature. In: SHANER, D.L., O'CONNOR, S.L. (Eds.). The imidazolinone herbicides, Boca Raton: CRC Press, 1991. p.191-209.

MICHAEL, J.L., NEARY, D.G. Herbicide dissipation studies in southern forest ecosystems. Environ. toxicol. chem., v.12, p.405-410, 1993.

MOSHIER, L.J., PENNER, D. Factors influencing microbial degradation of ${ }^{14} \mathrm{C}$-glyphosate to ${ }^{14} \mathrm{CO}_{2}$ in soil. Weed sci., v.26, n.6, p.686691, 1978.

NEWTON, M., HORNER, L.M., COWELL, J.E. et al. Dissipation of glyphosate and aminomethylphosphonic acid in North American forests. J. agric. food chem., v.42, n.8, p.1795-1802, 1994.

NICHOLLS, P.H. Factors influencing entry of pesticides into soil water. Pestic. sci., v.22, n.2, p.123-137, 1988.

PARKINSON, D., GRAY, T.R.G., WILLIAMS, S.T. Determination of microbial activity in soil. In: (Eds.). Methods for studying the ecology of soil micro- organisms. Oxford, England: Adlard \& Sons Ltd., 1971. p.71-101. (Handbook, 19)

PAUL, E.A., CLARK, F.E. Soil as a habitat for organisms and their reactions. In: (Eds.). Soil microbiology and biochemistry. San Diego, California: Academic Press, Inc., 1989. p.11-31.

PIPKE, R., AMRHEIN, N., JACOB, G.S. et al. Metabolism of glyphosate in an Arthrobacter sp. GLP-1. Eur. j. biochem., v.165, n.2, p.267-273, 1987.

PIVETZ, B.E., STEENHUIS, T.S. Soil matrix and macropore biodegradation of 2,4-D. J. environ. qual., v.24, n.4, p.564-570, 1995.

RAO, P.S.C., BELLIN, C.A., BRUSSEAU, M.L. Coupling biodegradation of organic chemicals to sorption and transport in soil and aquifers: paradigms and paradoxes. In: LINN, D.M., CARSKI, T.H., BRUSSEAU, M.L. et al. (Eds.). Sorption and degradation of pesticides and organic chemicals in soil. Madison, Wisconsin, USA: SSSA Inc., 1993. p.1-26. (SSSA Special publication, 32)

ROY, D.N., KONAR, S.K., BANERJEE, S. et al. Persistence, movement, and degradation of glyphosate in selected Canadian boreal forest soils. J. agric. food chem., v.37, n.2, p.437-440, 1989.

RUEPPEL, M.L., BRIGHTWELL, B.B., SHAEFER, J. et al. Metabolism and degradation of glyphosate in soil and water. J. agric. food chem., v.25, v.3, p.517-528, 1977.

SAKAMOTO, K., YOSHIDA, T. In Situ measurement of soil respiration rate by a dynamic method. Soil sci. plant nutr., v.34, n.2, p.195-202, 1988.

SKIPPER, H.D., WOLLUM II, A.G., TURCO, R.F. et al. Microbiological aspects of 
environmental fate studies of pesticides. Weed technol., v.10, n.1, p.174-190, 1996.

SMETS, B.F., JOBBÁGY, A., COWAN, R.M. et al. Evaluation of respirometric data: Identification of features that preclude data fitting with existing kinetic expressions. Ecotoxicol. environ. saf., v.33, n.1, p.8899, 1996.

SOUZA, A.P. de, LOURES, E.G., SILVA, J.F. da et al. Efeito do oxyfluorfen, 2,4-D e glyphosate na atividade microbiana de solos com diferentes texturas e conteúdos de matéria orgânica. Planta Daninha, v.14, n.1, p.55-64, 1996.

SPRANKLE, P., MEGGITT, W.F., PENNER, D. Adsorption, mobility, and microbial degradation of glyphosate in the soil. Weed sci., v.23, n.3, p.229-234, 1975.

TORSTENSSON, N.T.L., AAMISEPP, A. Detoxification of glyphosate in soil. Weed res., v.17, n.3, p.209-212, 1977.

TORSTENSSON, N.T.L., LUNDGREN, L.N., STENSTRÖM, J. Influence of climatic and edaphic factors on persistence of glyphosate and 2,4-d in forest soils. Ecotoxicol. environ. saf., v.18, n.3, p.230-239, 1989.

TORSTENSSON, N.T.L., STENSTRÖM, J. "Basic" respiration as a tool for prediction of pesticide persistence in soil. Toxic. ass., n.1, p.57-72, 1986.

VAN LOOSDRECHT, M.C.M., LYKLEMA, J., NORDE, W. et al. Influences of interfaces on microbial activity. Microbiol. rev., n.54, n.1, p.75-87, 1990.

WALKER, A. Herbicide persistence in soil. Rev. Weed sci., n.3, p.1-18, 1987.

WARDLE, D.A., NICHOLSON, K.S., RAHMAN, A. Influence of herbicide applications on the decomposition, microbial biomass, and microbial activity of pasture shoot and root litter. N. Z. j. agric. res., v.37, n.1, p.29-39, 1994.

WARDLE, D.A., PARKINSON, D. Effects of three herbicides on soil microbial biomass and activity. Plant and soil, v.122, n.1, p.21-28, 1990. 